moderate, 1 severe), while 6 (50.0\%) ApoE vessel segments had lesions (5 mild, 1 moderate). No lesions occurred in NZW animals. Two-tailed t-tests confirmed lesion occurrence more frequently in model animals $(p<0.001)$. There was no statistical difference in disease occurrence between WHHL and ApoE animals $(p=0.178)$. The observed presence of more advanced disease noted in WHHL rabbits compared to ApoE rabbits was not statistically significant $(p=0.296)$.

Conclusion ICAD can be reliably produced in rabbit models. Mature ApoE rabbits demonstrated less advanced ICAD after short term exposure to a hyperlipidemic diet compared to WHHL counterparts. Further analysis is needed to better characterize development and progression of disease in ApoE rabbits.

Disclosures M. Zabriskie: None. C. Wang: None. M. Alexander: None.

\section{E-020 SUSTAINABILITY IN INTERVENTIONAL RADIOLOGY: ARE WE DOING ENOUGH TO SAVE THE ENVIRONMENT?}

${ }^{1} \mathrm{P}$ Shum ${ }^{*},{ }^{2,3} \mathrm{H} \mathrm{Kok},{ }^{4,3} \mathrm{~J}$ Maingard, ${ }^{5,6} \mathrm{C}$ Barras, ${ }^{4,7} \mathrm{H}$ Asadi. ${ }^{1}$ Monash Health, Clayton, AUSTRALIA; ${ }^{2}$ Interventional Radiology Service, Department of Radiology, Northern Health, Epping, AUSTRALIA; ${ }^{3}$ School of Medicine, Faculty of Health, Deakin University, Geelong, AUSTRALIA; ${ }^{4}$ Interventional Neuroradiology Unit, Monash Imaging, Monash Health, Clayton, AUSTRALIA; ${ }^{5}$ Department of Radiology, Royal Adelaide Hospital, Adelaide, AUSTRALIA; ${ }^{6}$ South Australian Health and Medical Research Institute, Adelaide, AUSTRALIA; ' Interventional Neuroradiology Service, Department of Radiology, Austin Health, Heidelberg, AUSTRALIA

\subsection{6/neurintsurg-2020-SNIS.56}

Learning Objectives Global warming is a pressing issue. Healthcare waste contributes substantially to the world's carbon footprint. With IR playing an indispensable role in contemporary healthcare, the environmental impact of waste generated by IR procedures cannot be underestimated. The purpose of this literature review is 1) To identify waste generated and ways of reducing waste in Interventional Radiology (IR) practice. 2) To understand potential barriers of implementing green initiatives. 3) To quantify the environmental and financial impacts of waste generated and green initiatives. Materials and Methods A literature review was performed through electronic search of MEDLINE (PubMed, Ovid, SpringerLink and Wiley Online Library), ProQuest Nursing \& Allied Health Source and WorldCat.org databases using keywords 'radiology', 'interventional radiology', 'endovascular', 'recycling', 'waste', 'environment friendly', 'sustainability', 'greening', 'cost', 'climate change', 'global warming' and 'operating theatre'.

Results Greening initiatives include reducing, reusing and recycling waste, as well as strict waste segregation. Interventional radiologists can engage with suppliers to reformulate procedure packs to minimize unnecessary items and packaging. Opened, unused items can be prevented if there is better communication between doctors and support staff and increased awareness of wasted equipment cost amongst staff. Incentives to use soon-to-expire equipment can be offered. Power consumption can be reduced by powering down operating room lights and workstations when not in use, switching to light emitting diode (LED) and motion-activated lighting. Surgical hand wash can be replaced with alcohol-based hand rubs to reduce water usage. Common barriers to improving waste management include lack of leadership, misconception regarding infectious risk, lack of data, concerns about increased workload, negative staff attitudes and resistance to change. Education remains a top priority to engage all staff in sustainable healthcare practices.

Conclusion Interventional radiologists have a crucial role to make healthcare more sustainable. By making small changes in our practices, rewarding results in terms of financial savings and environmental sustainability can be achieved.

Disclosures P. Shum: None. H. Kok: 2; C; Northern Health. J. Maingard: None. C. Barras: 2; C; Royal Adelaide Hospital. H. Asadi: 2; C; Austin Health, Monash Health.

\section{E-021 EFFECT OF EARLY SANGUINATE ${ }^{\oplus}$ INFUSION ON CEREBRAL BLOOD FLOW TO THE ISCHEMIC CORE IN EXPERIMENTAL MIDDLE CEREBRAL ARTERY OCCLUSION}

${ }^{1} \mathrm{G}$ Christoforidis*, ${ }^{1} \mathrm{~N}$ Saadat, ${ }^{2} \mathrm{M}$ Niekrasz, ${ }^{3} \mathrm{~S}$ Roth, ${ }^{1} \mathrm{~T}$ Carroll. ${ }^{1}$ Radiology, University of Chicago, Chicago, IL; ${ }^{2}$ Surgery, University of Chicago, Chicago, IL; ${ }^{3}$ Anesthesiology, University of Illinois at Chicago, Chicago, IL

10.1136/neurintsurg-2020-SNIS.57

Purpose Sanguinate (Prolong Pharmaceuticals, South Plainfield, NJ) is an investigational bio-pharmaceutical blood substitute that facilitates the transfer of oxygen to oxygendeprived cells and tissues using a bovine PEGylated carboxyhemoglobin-based oxygen carrier designed to release oxygen in hypoxic tissue. Through its vasodilatory effects at the pial arteriolar level, its ability to deliver oxygen to severely hypoxic tissues, oncotic effect and anti-inflammatory effects, Sanguinate shows promise in slowing the progression of ischemic insult in acute stroke. We hypothesize that Sanguinate improves cerebral blood flow to the ischemic core in the early stages of MCA occlusion (MCAO).

Methods Using an IACUC approved protocol, eight mongrel canines (20-30 kg) underwent angiographically-guided endovascular permanent MCA occlusion. Four subjects received Sanguinate $(8 \mathrm{~mL} / \mathrm{kg})$ starting 30 minutes following MCA occlusion and four subjects received a similar volume of normal saline (controls). Physiologic parameters $\mathrm{pH}, \mathrm{PaO}_{2}$, $\mathrm{PaCO}_{2}$, glucose, hematocrit, mean arterial blood pressure, $\mathrm{ETCO}_{2}$, temperature and glucose were monitored and kept within physiologic limits. Anesthetics were chosen to minimize cerebrovascular effects. Cerebral blood flow (CBF) was assessed using neutron-activated microspheres (Biopal, Inc, Worcester, MA, USA) at three time points: 1) prior to MCAO, 2) 30 minutes following MCAO and 3) 30 minutes following Sanguinate/saline infusion. Pial collateral recruitment was scored and measured by arterial arrival time 30 minutes post MCAO (immediately prior to Sanguinate/saline infusion) and 30 minutes post Sanguinate/saline infusion. Diffusionweighted MRI (3T Achieva, Philips Healthcare, Best, Netherlands) was used to assess infarct volume 2 hours following MCAO. CBF values, infarct volumes and arterial arrival time in the control and treatment groups were compared using one-way analysis of variance assuming equal variances with significance defined at the 5\% level.

Results Mean infarct volumes two hours following MCAO for control and Sanguinate treated subjects were $2122 \mathrm{~mm}^{3}$ and $2440 \mathrm{~mm}^{3}(\mathrm{p}=0.8173)$, respectively. Relative to pre-treatment post-MCAO CBF, CBF measured 30 minutes following Sanguinate infusion increased by a mean of $25.2 \%$ whereas in controls CBF increased by a mean of $1.44 \%$ following saline infusion $(p=0.0270$; figure 1$)$. Pre-treatment pial collateral recruitment between treatment and control groups were nearly 